\title{
Dynamic Measurement of Relative Complex Permittivity of Microwave Plasma at Atmospheric Pressure
}

\author{
Ge Wang, Hui Pan, Shimiao Lai, Yongjie Zhou, Li Wu, Huacheng Zhu $\mathbb{D}$ and Yang Yang * \\ College of Electronic and Information Engineering, Sichuan University, Chengdu 610065, China;

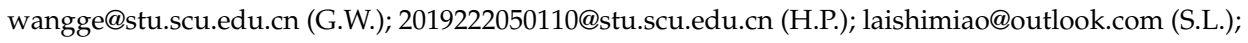 \\ zzzhouyongjie@163.com (Y.Z.); wuliscu@126.com (L.W.); hczhu@scu.edu.cn (H.Z.) \\ * Correspondence: yyang@scu.edu.cn; Tel.: +86-28-8547-0659
}

check for updates

Citation: Wang, G.; Pan, H.; Lai, S.; Zhou, Y.; Wu, L.; Zhu, H.; Yang, Y. Dynamic Measurement of Relative Complex Permittivity of Microwave Plasma at Atmospheric Pressure. Processes 2021, 9, 1812. https:// doi.org/10.3390/pr9101812

Academic Editor: Jean-Pierre Corriou

Received: 6 September 2021

Accepted: 9 October 2021

Published: 13 October 2021

Publisher's Note: MDPI stays neutral with regard to jurisdictional claims in published maps and institutional affiliations.

Copyright: (C) 2021 by the authors Licensee MDPI, Basel, Switzerland. This article is an open access article distributed under the terms and conditions of the Creative Commons Attribution (CC BY) license (https:/ / creativecommons.org/licenses/by/ $4.0 /)$.

\begin{abstract}
Complex permittivity is one of the most important parameters to characterize the interaction between microwave and medium, especially for microwave-excited plasma. It is convenient to study plasma's dielectric properties and microwave propagation characteristics by measuring its complex permittivity. A dynamic measurement method of equivalent relative complex permittivity of microwave-excited plasma at atmospheric pressure is proposed in this paper. Firstly, a cavity based on WR-430 at a frequency of $2.45 \mathrm{GHz}$ was specially designed in COMSOL. Then, the samples with different real parts of complex permittivity and loss tangent were simulated in the designed cavity to obtain their corresponding S parameters, and they were used to train the BP neural network until the error was lower than 0.001. A two-port network was built to excite the plasma. The input power, reflected power, and transmitted power could be measured by the transmission reflection method. Finally, the measured power values were converted into $S$ parameters and used as inputs in the BP neural network. The plasma's real parts of complex permittivity and loss tangent were obtained by inversion. The variation of plasma complex permittivity conforms to the interaction principles between microwave and plasma, which verifies the accuracy of the method.
\end{abstract}

Keywords: microwave plasma; complex permittivity; BP neural network

\section{Introduction}

Generally, plasma can be equivalent to a medium whose dielectric properties are characterized by equivalent complex permittivity. By measuring the complex permittivity of the medium, the design of materials and cavity can be optimized in the simulation, which is of great significance to improve the utilization efficiency of microwave. Therefore, complex permittivity measurement has been applied in various fields such as aerospace and industry [1,2]. In recent years, some novel metamaterials and plasma with distinctive dielectric properties to those of conventional materials have emerged. The real part of their complex permittivity is negative [3] with a wide range, which fluctuates with the microwave frequency, power, temperature and pressure. So, it poses a new challenge to the measurement of complex permittivity.

The method of measuring complex permittivity $[4,5]$ is composed of the resonance method [6,7] and network parameter method [8,9]. These methods have achieved remarkable results in complex permittivity measurement of conventional materials [10-13], but there were few reports on the complex permittivity measurement of microwave plasma.

Currently, the complex permittivity of plasma is mostly obtained by theoretical calculation. Jiansheng Liu [14] gave effective dielectric constant of plasma by solving Maxwell's equation. Felderhof [15] used linear response theory and the technique of cluster expansion to study the dielectric function of plasma by quasistatic approximation, but it is no longer applicable when the electron temperature and the density of plasma change rapidly. Mascali et al. [16] used a Langmuir probe to measure the electron density and other plasma parameters, and they obtained the equivalent relative complex permittivity of plasma by 
calculation. However, when some plasma domains do not allow the probe to penetrate, this method is no longer applicable.

The interaction between microwave and plasma is complex. Dynamic measurement of the complex permittivity of plasma is helpful to observe the interaction principles between microwave and plasma under different conditions. Therefore, a dynamic measurement method of the complex permittivity of microwave plasma is proposed in this paper. Firstly, in order to build a two-port network to excite plasma, we designed a cavity based on WR-430 in COMSOL. We simulated the samples with different complex permittivity to obtain the corresponding $\left|S_{11}\right|$ and $\left|S_{21}\right|$ parameters in the designed cavity, and they were used to train a BP neural network [17]. Then, we measured the input power, reflected power, and transmitted power by the transmission reflection method $[18,19]$ in the two-port network. Multiple groups of reflected power and transmitted power could be obtained by changing the gas flow and microwave power. Finally, the measured power was converted into $\left|S_{11}\right|$ and $\left|S_{21}\right|$ parameters, and they were used as inputs to obtain the complex permittivity of plasma by BP neural network inversion. We have drawn and analyzed the curve of the microwave plasma's real part of complex permittivity and loss tangent with different input power and gas flow.

\section{Methodology}

\subsection{Schematic Design}

The two-port network measurement system based on the transmission reflection method is shown in Figure 1. Argon is excited by microwave to form plasma in the center of a quartz tube. The power meter close to the microwave source measures the input power $\left(\mathrm{P}_{1}\right)$ and the reflected power $\left(\mathrm{P}_{2}\right)$, and another power meter measures the transmitted power $\left(\mathrm{P}_{3}\right)$. The measured power will be converted into $\left|\mathrm{S}_{11}\right|$ and $\left|\mathrm{S}_{21}\right|$ parameters, and they are used as the inputs of the trained BP neural network to obtain the plasma's real part of complex permittivity and loss tangent by inversion. The flowchart is shown in Figure 2. Moreover, in this measurement system, we realize the dynamic measurement of the plasma complex permittivity when changing the microwave power and gas flow.

\subsection{Cavity Design}

We designed an open-end cavity based on WR-430. The cut-off waveguide in the center of the cavity is to prevent microwave power from leaking out. In order to give the center of the cavity high electric field intensity to excite the plasma and improve the transmission performance of the cavity, the length of the cavity as well as the length and inner diameter of the cut-off waveguide were scanned parametrically in COMSOL. Finally, the cavity structure is determined and processed, as shown in Figure 3.

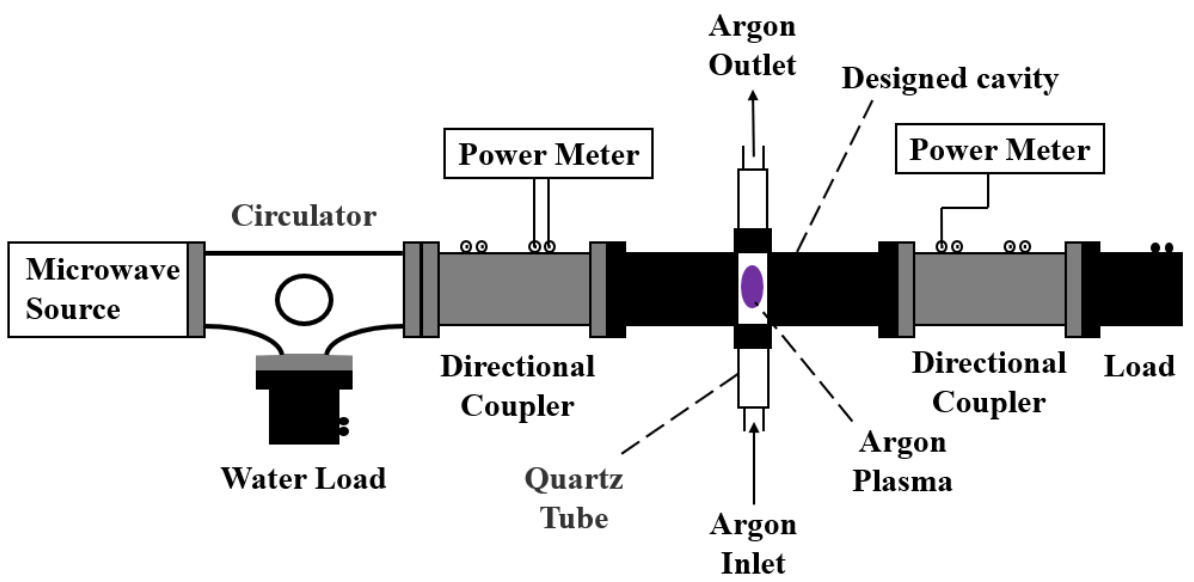

Figure 1. The schematic diagram of the device. 


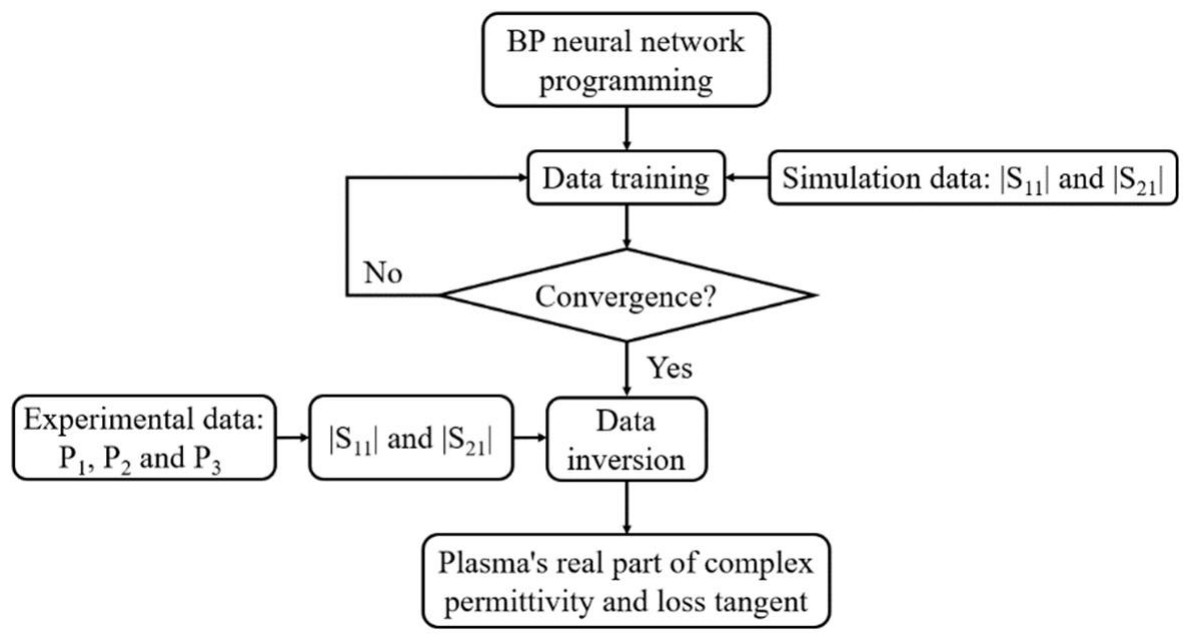

Figure 2. The scheme flowchart.

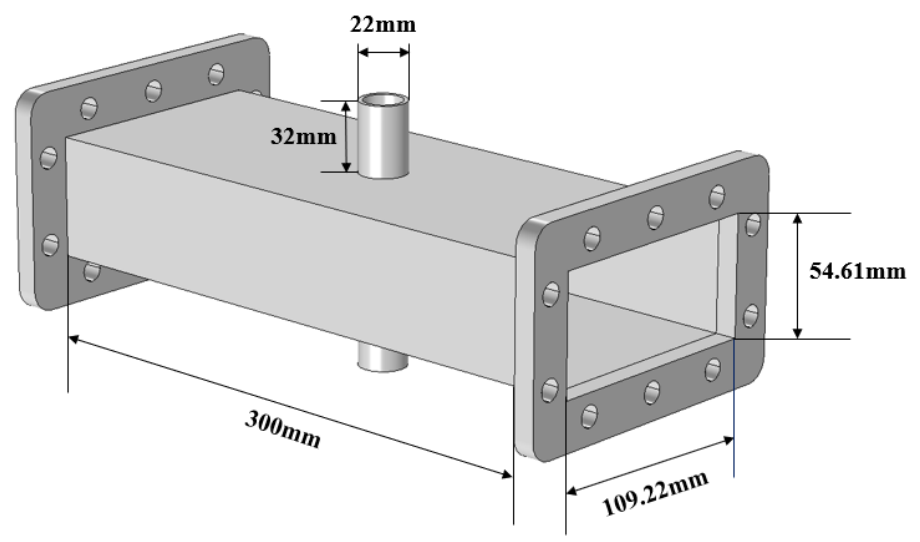

Figure 3. Schematic of cavity.

A quartz tube with a wall thickness of $2 \mathrm{~mm}$ is placed in the cut-off waveguide to excite the plasma. It is assumed that the plasma in the quartz tube is homogenously distributed. Therefore, the complex permittivity of the samples set in COMSOL is regarded as the equivalent complex permittivity of the plasma. We can obtain the $S$ parameters corresponding to different samples by simulation. Considering the large variation range of plasma complex permittivity and high loss, the samples' range and step of the complex permittivity real part and the loss tangent are set in the parameter scanning, as shown in Table 1. Figure 4 shows the simulation results. Within the parameter setting range, the $\left|S_{11}\right|$ and $\left|S_{21}\right|$ parameters of the port maintain good monotonicity, which can effectively avoid a multi-value problem in BP neural network inversion, so as to obtain more accurate results.

Table 1. Parameter scan settings.

\begin{tabular}{cc}
\hline Real part range & $-200-0$ \\
\hline Real part step & 1 \\
\hline Loss tangent range & $-1-0$ \\
\hline Loss tangent step & 0.1 \\
\hline
\end{tabular}




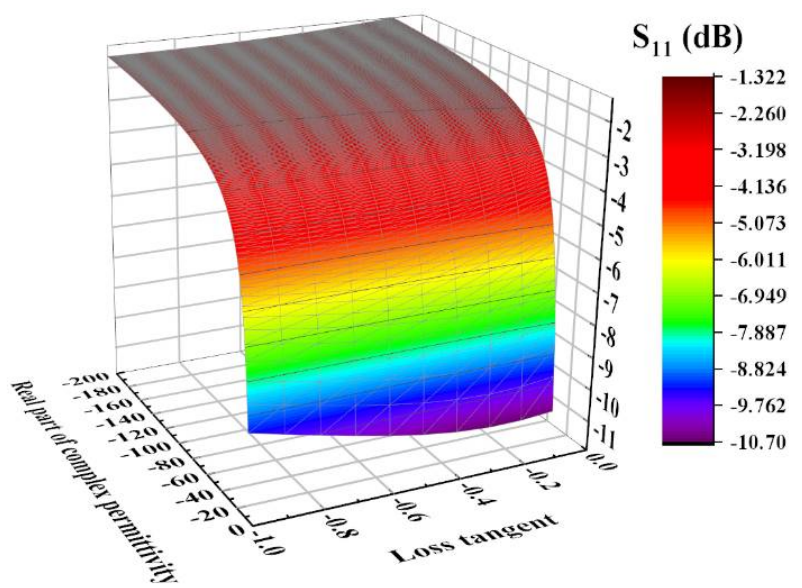

(a) Amplitude of $S_{11}$

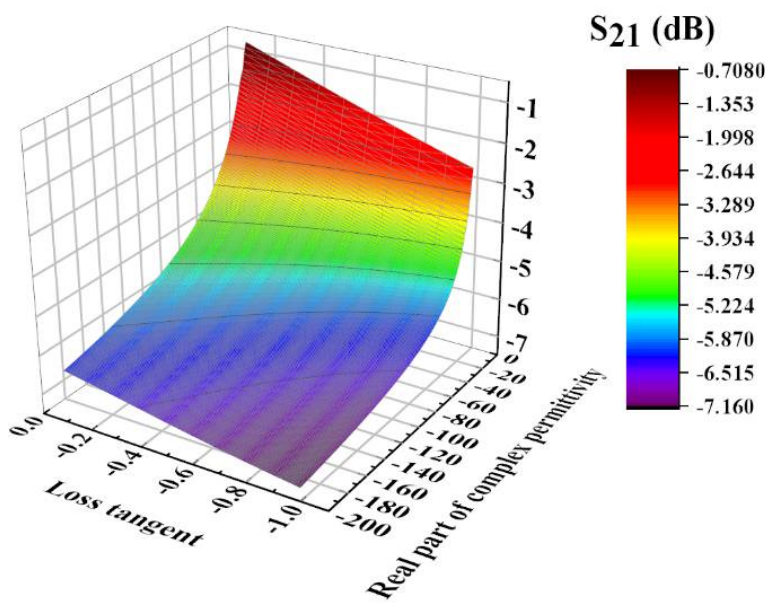

(b) Amplitude of $S_{21}$

Figure 4. Simulation results.

\subsection{BP Neural Network}

In this paper, BP neural network is used to inverse the dielectric properties of plasma. $\mathrm{BP}$ neural network is a multilayer feedforward network trained according to the error back propagation algorithm. It can learn and store a large number of input-output mode mapping relationships without describing the mathematical equations of this mapping relationship. The process is mainly divided into two stages. The first stage is the forward propagation of the signal from the input layer to the hidden layer and finally to the output layer. The second stage is the back propagation of the error from the output layer to the hidden layer and finally to the input layer. By the means of back propagation, the weight and threshold of the network are continuously adjusted to minimize the sum of squares of the error of the network. BP neural network is currently one of the most widely used neural network models.

Since the neural network algorithm can accurately inverse the mapping relationship between input values and output values only when they are corresponding, it is necessary to discuss the multi-value problem of the neural network before taking the value of the sample space. It is known from Figure 4 that the corresponding relationship between input values and output values maintains monotonicity. The two-input and two-output network structure can greatly reduce the multi-value points and effectively avoid the multi-value problem. This paper constructs a BP neural network with $\left|S_{11}\right|$ and $\left|S_{21}\right|$ parameters as inputs and the real part of complex permittivity and loss tangent as outputs, as shown in Figure 5.

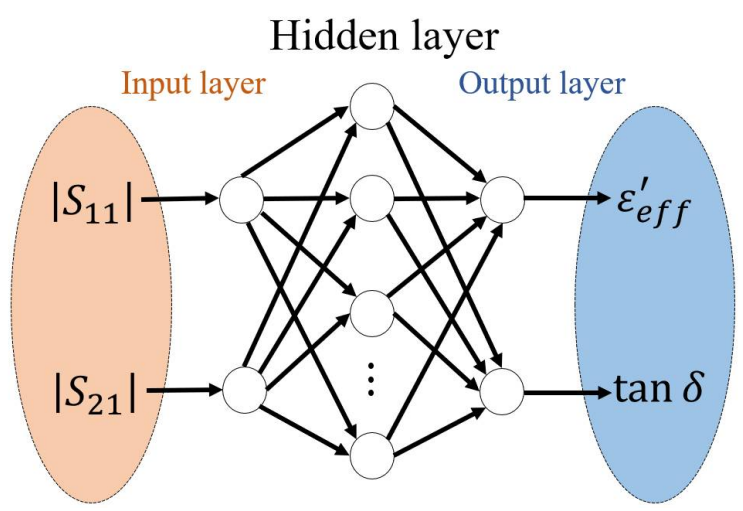

Figure 5. BP neural network structure. 
The simulation results are randomly assigned to one of two sets of samples: $80 \%$ to the training set and 20\% to the test set. Furthermore, the BP neural network training parameters are shown in Table 2.

Table 2. Parameters of BP neural network.

\begin{tabular}{ccccc}
\hline Study Radio & $\begin{array}{c}\text { Maximum Iteration } \\
\text { Times }\end{array}$ & $\begin{array}{c}\text { Target } \\
\text { Accuracy }\end{array}$ & $\begin{array}{c}\text { Hidden } \\
\text { Neuron }\end{array}$ & $\begin{array}{c}\text { Hidden } \\
\text { Layers }\end{array}$ \\
\hline 0.035 & 3000 & 0.001 & 10 & 1 \\
\hline
\end{tabular}

\subsection{Experimental System}

The measuring system is mainly composed of a microwave source (WSPS-2450500TW, Wattsine, Chengdu, China), circulator (ELCLWG2.45-0.1P1K, Euler Microwave, Chengdu, Sichuan Province, China), water load (TMWG26WLA10P3KW, Euler Microwave, Chengdu, Sichuan Province, China), power meter (AV2433, the 41st Institute of China Electronic Technology Group Corporation, Hefei, Anhui Province, China), directional coupler (LOOP2450B100C40A10N/LOOPE22DC40A10N, Euler Microwave, Sichuan Province, China), and flowmeter (MF5706, Nanjing, Jiangsu Province, China). The measurement system is shown in Figure 6a.

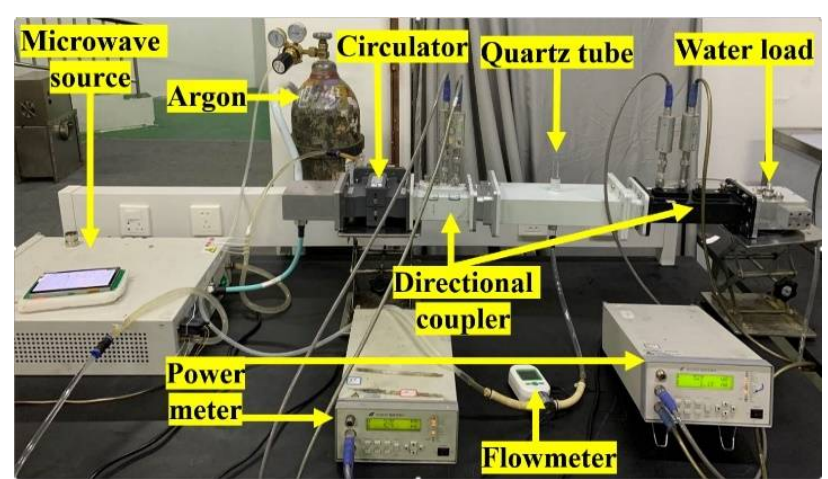

(a)Experiment system

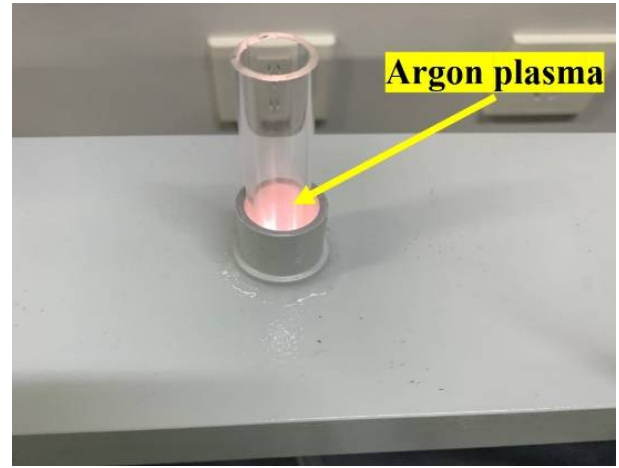

(b)Microwave-excited plasma

Figure 6. Measurement system.

The microwave source can be adjusted to different values of power, up to $500 \mathrm{~W}$. The circulator prevents excessive reflected power from damaging the microwave source. The water load absorbs excess energy. The quartz tube is placed in the center of the designed cavity. The flowmeter is connected to the lower end of the quartz tube to adjust the gas flow. When the microwave excites argon gas to generate plasma, as shown in Figure 6b, the input power, reflected power, and transmitted power from the microwave source to plasma can be measured by the power meter. Then, we adjusted the different power and gas flow to get the corresponding $S$ parameters.

\section{Measured Results and Discussion}

Argon is introduced into the bottom of the quartz tube, and the initial gas flow is set to $10 \mathrm{~L} / \mathrm{min}$. When the gas flow is stable, the microwave source is turned on, and the microwave power is initially set to $150 \mathrm{~W}$, which is the minimum power to excite argon plasma in this measurement system. Then, we gradually increased the power and gas flow when the plasma was excited in the middle of the quartz tube. The range and step are shown in Table 3. The reflected power and transmitted power under different input power and gas flow are recorded, and the measurement error of the power meter is within $10 \%$. The measured power is converted to the corresponding $\mathrm{S}$ parameters, as shown in Figure 7. Taking the $\left|S_{11}\right|$ and $\left|S_{21}\right|$ parameters as inputs, the real part of the plasma complex permittivity and loss tangent corresponding to different power and gas flow are 
obtained by means of BP neural network inversion, as shown in Figure 8. Moreover, the inversion error of the BP neural network is within $20 \%$.

Table 3. Power and gas flow parameters.

\begin{tabular}{cccc}
\hline & Start & End & Step \\
\hline Power & $150 \mathrm{~W}$ & $500 \mathrm{~W}$ & $50 \mathrm{~W}$ \\
Gas Flow & $10 \mathrm{~L} / \mathrm{min}$ & $25 \mathrm{~L} / \mathrm{min}$ & $5 \mathrm{~L} / \mathrm{min}$ \\
\hline
\end{tabular}

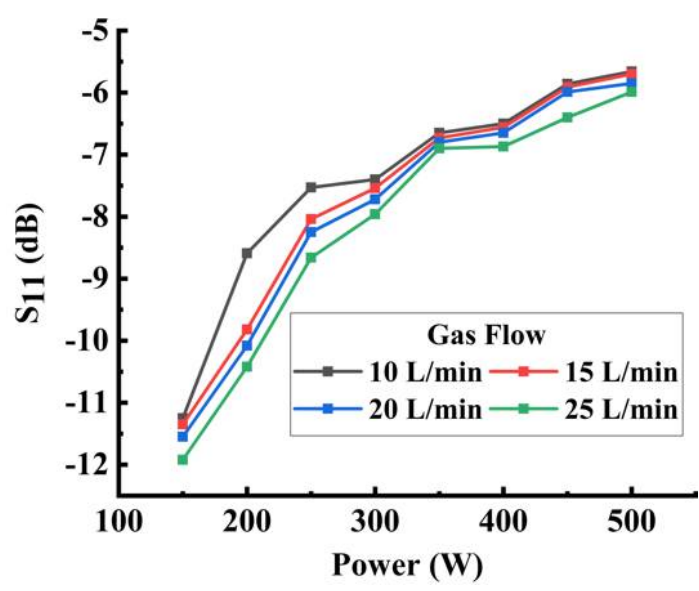

(a)S11 curve

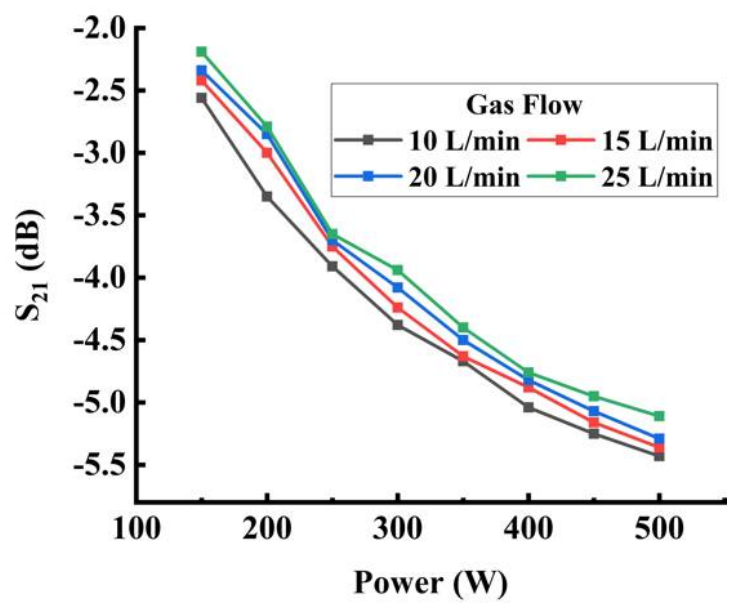

(b) $S_{21}$ curve

Figure 7. S parameters corresponding to different power and gas flow.
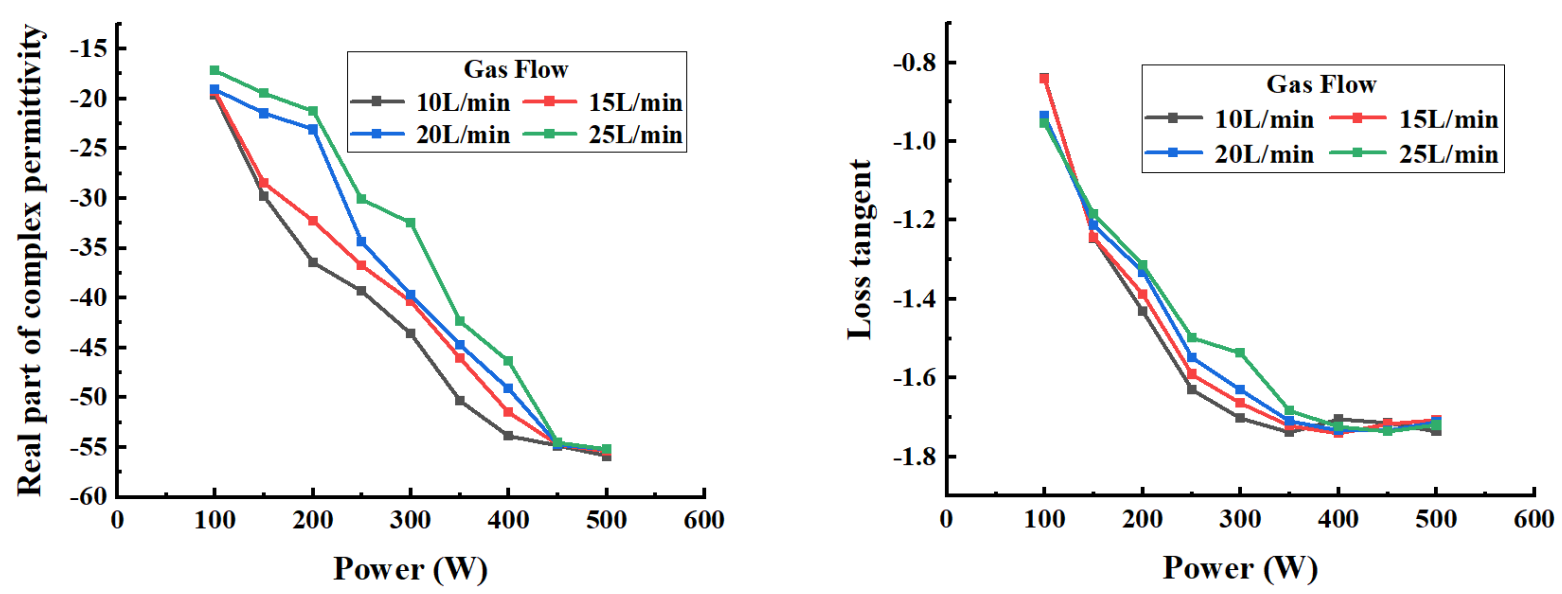

Figure 8. Real part of complex permittivity and loss tangent corresponding to different power and gas flow.

Figure $7 \mathrm{a}, \mathrm{b}$ show that $\left|S_{11}\right|$ increases and $\left|S_{21}\right|$ decreases with the rise of power. This is due to the fact that with lower input power, the excited plasma electron density becomes lower accordingly, which makes the microwave easier to penetrate plasma, causing less reflection. With higher input power, the excited plasma electron temperature becomes higher and the electron collision frequency increases, which leads to higher electron density. With the increase in electron density, the ability of plasma to reflect microwave energy is stronger, and the attenuation of microwave energy is greater [20]. When the power reaches $500 \mathrm{~W},\left|S_{11}\right|$ reaches its maximum and $\left|S_{21}\right|$ reaches its minimum.

Figure $8 \mathrm{a}, \mathrm{b}$ show that the real part of the complex permittivity of plasma decreases from -15 to -55 and the loss tangent decreases from -0.8 to -1.8 in the power range from 150 to $450 \mathrm{~W}$. Then, they stay stable in the power range from 450 to $500 \mathrm{~W}$. Due to reflection, the real part of plasma's complex permittivity is negative. With the rise of power, 
the reflection increases, and the real part of plasma complex permittivity decreases. When the power reaches $450 \mathrm{~W}$, the electron density and electron collision frequency of plasma reach the maximum and then stay stable. At this time, microwave energy cannot penetrate the inside of the plasma [21]. The loss of microwave energy no longer fluctuates with the increase in power.

Keeping the input power constant and increasing the gas flow, the plasma will be blown to the far end of the quartz tube. Under such circumstance, electron loss might occur. Therefore, the electron density decreases in the tube, which makes it easy for the microwave power to penetrate with low reflection. Moreover, under the same power level, larger gas flow makes smaller $\left|S_{11}\right|$, larger $\left|S_{21}\right|$, and complex permittivity. However, when the power is large enough and plasma reaches a stable state, changing the gas flow will no longer affect the real part of the complex permittivity and loss tangent.

\section{Conclusions}

From a macroscopic perspective, complex permittivity is an important parameter representing the interaction of plasma excited by microwave. In this paper, a dynamic measurement method of microwave plasma relative complex permittivity at atmospheric pressure is proposed to better study the variation of plasma complex permittivity against microwave power and gas flow. Firstly, an experimental cavity based on WR-430 was designed. COMSOL Multiphysics was used to simulate the samples represented by a wide range of complex permittivity to obtain their corresponding $\left|S_{11}\right|$ and $\left|S_{21}\right|$ parameters. Then, the simulation data were used as the training set to construct a BP neural network. The $\left|S_{11}\right|$ and $\left|S_{21}\right|$ parameters were used as inputs, and the real parts of complex permittivity and loss tangent were used as outputs. In the experiment, by controlling the gas flow and microwave source, the input, reflected, and transmitted power were measured, which were later converted into $\left|S_{11}\right|$ and $\left|S_{21}\right|$ parameters. By means of a BP neural network inversion algorithm, the corresponding real parts of complex permittivity and loss tangent were obtained. Compared with the traditional method, the proposed method is capable of real-time measurement of plasma complex permittivity under varying external conditions. Compared with traditional inversion algorithms, the BP neural network has strong nonlinear mapping ability, and the inversion results are fast and accurate. Compared with related theories, the measured plasma complex permittivity curve conforms to theoretical reaction principles, which proves the feasibility and accuracy of the proposed method. In the simulation of microwave and argon plasma, this paper provides reference values for the complex permittivity of argon plasma. The method also provides a new reference for the measurement of medium with a large range of complex permittivity.

Author Contributions: G.W. developed the model, finished the experiment, analyzed the data, and wrote the initial draft of the manuscript; H.P., S.L. and Y.Z. conceived and designed the experiment; L.W., H.Z. and Y.Y. reviewed and contributed to the final manuscript. All authors have read and agreed to the published version of the manuscript.

Funding: This work was supported by the Sichuan Science and Technology Program under Grant 2021YFG0265, the Sichuan University and Panzhihua City project (Grant No. 2019CDPZH-20), and the Sichuan University and Suining City project (Grant No. 2020CDSN-01).

Institutional Review Board Statement: Not applicable.

Informed Consent Statement: Not applicable.

Conflicts of Interest: The authors declare no conflict of interest.

\section{References}

1. Gershon, D.L.; Calame, J.P.; Carmel, Y.; Antonsen, T.M.; Hutcheon, R.M. Open-ended coaxial probe for high-temperature and broad-band dielectric measurements. IEEE Trans. Microw. Theory Tech. 1999, 47, 1640-1648. [CrossRef]

2. Yang, X.; Huang, K. The empirical formula for calculating the complex effective permittivity of an aqueous electrolyte solution at microwave frequency. IEEE Trans. Geosci. Remote Sens. 2005, 43, 315-320. [CrossRef] 
3. Karpman, V.I. High frequency electromagnetic field in plasma with negative dielectric constant. Plasma Phys. 1971, 13, 477-490. [CrossRef]

4. Severo, S.L.; de Salles, Á.A.; Nervis, B.; Zanini, B.K. Non-resonant Permittivity Measurement Methods. J. Microw. Optoelectron. Electromagn. Appl. 2017, 16, 297-311. [CrossRef]

5. Hickson, D.; Sotodeh, S.; Daly, M.G.; Ghent, R.; Nolan, M.C. Improvements on effective permittivity measurements of powdered alumina: Implications for bulk permittivity properties of asteroid regoliths. Adv. Space Res. 2016, 59, 472-482. [CrossRef]

6. Santra, M.; Limaye, K.U. Estimation of complex permittivity of arbitrary shape and size dielectric samples using cavity measurement technique at microwave frequencies. IEEE Trans. Microw. Theory Tech. 2005, 53, 718-722. [CrossRef]

7. Sten, C.E.; Koivisto, P.K. TECHNICAL DESIGN NOTE: Determining both the permittivity and the permeability of small samples using cavity perturbation method. Meas. Sci. Technol. 2009, 20, 118-122. [CrossRef]

8. Janezic, M.D.; Williams, D.F. Permittivity Characterization from Transmission-Line Measurement. In Proceedings of the 1997 IEEE MTT-S International Microwave Symposium Digest, Denver, CO, USA, 8-13 June 1997; pp. 1343-1346.

9. Hosseini, M.H.; Heidar, H.; Shams, M.H. Wideband Nondestructive Measurement of Complex Permittivity and Permeability Using Coupled Coaxial Probes. IEEE Trans. Instrum. Meas. 2016, 66, 148-157. [CrossRef]

10. Inoue, R.; Odate, Y.; Tanabe, E.; Kitano, H.; Maeda, A. Data analysis of the extraction of dielectric properties from insulating substrates utilizing the evanescent perturbation method. IEEE Trans. Microw. Theory Tech. 2006, 54, 522-532. [CrossRef]

11. Jha, A.K.; Akhtar, M.J. A Generalized Rectangular Cavity Approach for Determination of Complex Permittivity of Materials. IEEE Trans. Instrum. Meas. 2014, 63, 2632-2641. [CrossRef]

12. Chen, Q.; Yang, Y.; Huang, K.M.; Chen, C.; Wang, K.Y. A Permittivity Measurement Method Based on Cavity Perturbation Technique. Appl. Mech. Mater. 2014, 590, 629-633. [CrossRef]

13. Ghodgaonkar, D.K.; Varadan, V.V.; Varadan, V.K. Free-space measurement of complex permittivity and complex permeability of magnetic materials at microwave frequencies. IEEE Trans. Instrum. Meas. 1990, 39, 387-394. [CrossRef]

14. Liu, J.; Li, R.; Zhu, P.; Zhu, Y.; Xu, Z. Strong interaction of rare gas clusters with high-intensity laser pulses. In Proceedings of the 4th Pacific Rim Conference on Lasers and Electro-Optics, Chiba, Japan, 15-19 July 2001.

15. Felderhof, B.U.; Beński, T.; Cichocki, B. Dielectric function of an electron-ion plasma in the optical and X-ray regime. Phys. A 1995, 217, 161-174. [CrossRef]

16. Mascali, D.; Gambino, N.; Miracoli, R.; Gammino, S.; Torrisi, L.; Maimone, F.; Tumino, L. Plasma parameters measurements by means of Langmuir probe. Radiat. Eff. Defects Solids 2008, 163, 471-478. [CrossRef]

17. Sadeghi, B. A BP-neural network predictor model for plastic injection molding process. J. Mater. Process. Technol. 2000, 103, 411-416. [CrossRef]

18. Nicolson, A.M.; Ross, G.F. Measurement of the Intrinsic Properties of Materials by Time-Domain Techniques. IEEE Trans. Instrum. Meas. 1970, 19, 377-382. [CrossRef]

19. Hasar, U.C. A Fast and Accurate Amplitude-Only Transmission-Reflection Method for Complex Permittivity Determination of Lossy Materials. IEEE Trans. Microw. Theory Tech. 2008, 56, 2129-2135. [CrossRef]

20. Hagelaar, G.; Hassouni, K.; Gicquel, A. Interaction between the electromagnetic fields and the plasma in a microwave plasma reactor. J. Appl. Phys. 2004, 96, 1819-1828. [CrossRef]

21. Sakai, O.; Iwai, A.; Omura, Y.; Iio, S.; Naito, T. Wave propagation in and around negative-dielectric-constant discharge plasma. Phys. Plasmas 2018, 25, 031901. [CrossRef] 